\title{
Toward Texture-Based 3D Level Set Image Segmentation
}

\author{
Daniel Reska, Cezary Boldak and Marek Kretowski
}

\begin{abstract}
This paper presents a three-dimensional level set-based image segmentation method. Instead of the typical image features, like intensity or edge information, the method uses texture feature analysis in order to be more applicable to image sets withs distinctive patterns. The current implementation makes use of a set of Grey Level Co-occurrence Matrix texture features that are generated and selected according to the characteristics of the initial region. The region is then deformed using the level set-based algorithm to cover the desired image area. The generation of the texture features and the level set surface deformation scheme are performed with graphics card hardware acceleration. The preliminary experiments, performed on synthetic data sets, show promising segmentation results.
\end{abstract}

Keywords Image segmentation $\cdot$ Deformable models $\cdot$ Level set $\cdot$ Texture analysis

\section{Introduction}

Level set methods are a group of algorithms broadly used in many areas of computer science, particularly in computer vision [14]. Level set-based deformable models [7] are especially useful for the task of image segmentation, i.e. partitioning of the image into distinct regions. The idea of a level set segmentation is based on an implicitly represented shape (a curve for a 2D image or a surface for a 3D volume), defined within the image domain. This shape is then deformed in an evolution process driven by external (image data) and internal (smoothness/curvature) forces. The resulting shape contains the targeted image region of desired characteristics.

D. Reska $(\bowtie) \cdot$ C. Boldak $\cdot$ M. Kretowski

Bialystok University of Technology, Bialystok, Poland

e-mail: d.reska@pb.edu.pl

C. Boldak

e-mail: c.boldak@pb.edu.pl

M. Kretowski

e-mail: m.kretowski@pb.edu.pl 
Level sets have an important ability to adapt their topology, which is a big advantage over the traditional parametric deformable models [4]. A level set shape can contract or expand, but also naturally divide or merge, therefore can handle changes in the segmented region topology without any additional frameworks [6]. The image data forces can be also modified to incorporate region [2, 13] or texture-based [9, 15] features. A known downside of the level sets is their computational intensity, especially in 3D. Recently, this limitation has been addressed by utilization of the graphics card (GPU) acceleration, which can significantly increase the performance of the 3D methods $[5,12]$ and open new possibilities for more interactive applications.

In this paper, we propose a texture-based level set method for segmentation of three-dimensional image volumes. The method replaces the traditional intensitybased data term in the level set speed equation with a term based on image texture features. In its current form, the method utilizes features based on Gray-Level Cooccurrence Matrices (GLCM) [3]. The texture feature generation process, as well as the level set evolution algorithm, uses hardware-accelerated implementations on GPU. The preliminary experiments were performed on synthetic images, where the texture-based approach showed an improvement over traditional methods.

\section{Texture-Based Level Set Method}

The proposed segmentation method incorporates a texture-based image data term into a 3D level set algorithm. In the image domain, the level set defines a surface that can deform according to the characteristics of the initial region: the surface can expand into a region with a uniform texture that is similar to the initial region or contract from an area where the texture does not meet the similarity criteria.

The initial spherical surface is manually initialized inside the segmented region. The texture features are then generated for the part of the volume inside the bounding box of the surface. Next, the algorithm selects the most uniform features for the initial region and uses them in the level set-based deformation process.

\subsection{Texture Feature Generation and Selection}

The texture feature generation process creates a set of feature maps for the volume inside the initial surface and then selects the features that have a low dispersion inside the initial region. This condition selects the texture features that will distinguish the segmented area from regions with different textures.

Currently, the method uses 2D texture features, generated separately for each of the images in the data stack (that composes the volume). To find the initial texture feature set, the algorithm selects the $2 \mathrm{D}$ images intersected by the bounding box of the initial surface and calculates partial texture maps only for the areas of the 
intersection. A mask of the initial surface region is also generated and used to calculate the characteristics of the features only for the volume points (voxels) inside the start region. For each of the features $m_{i}$, the mean of the feature values $\bar{x}_{i}$, standard deviation $\sigma_{i}$ and Relative Standard Deviation $\% R S D_{i}=\frac{\sigma_{i}}{\bar{x}_{i}} \times 100$ are calculated. The texture feature used for the segmentation process must have the $\% R S D$ lower than a user-specified threshold (equal to $65 \%$ by default). After this selection step, all of the selected features are calculated for the entire input data set (again in 2D: each selected feature for every image). Additionally, the selection step can reduce the number of maps by analyzing their similarity for different orientations in groups with the same feature, window size and displacement. The details of this process are described in [10].

The currently used texture features, generated from the Grey-Level Co-occurrence Matrix, are: Entropy, Correlation, Homogeneity, Contrast and Energy. The maps are generated for different sets of GLCM parameters: window size (from $3 \times 3$ to $11 \times 11$ by default), displacement (from 1 to 3 pixels) and orientation $\left(0^{\circ}, 45^{\circ}, 90^{\circ}, 135^{\circ}\right.$ and for all four angles). The algorithm, however, is not limited to the GLCM approach any method that can generate a feature map of the segmented image can be used.

\subsection{Level Set Surface Deformation}

The manually initialized surface is deformed using the level set method. In this approach, we can define the surface $S$ in a form of a set of points $p=(x, y, z)$ in the image domain as $S=\{p \mid \phi(p, t)=0\}$, where $\phi(p, t): \mathfrak{R}^{3} \mapsto \mathfrak{R}$ and $t$ is the time step of the simulation. During the simulation process, the surface points are moved according to a speed function $F(p, t)$, which allows the surface to expand or contract in order to eventually enclose the segmented region.

In this work, we modify the speed function proposed by Lefohn et al. [5], defined as:

$$
F(p, t)=\alpha D(p)+(1-\alpha) C(p, t),
$$

where $D(p)$ is the image data term that drives the deformation, $C(p, t)$ is the surface curvature term and $\alpha \in[0,1]$ is a user-defined parameter for balancing the influence of the two terms. The original image intensity-based data term was defined as:

$$
D_{\text {int }}(p)=\epsilon-|I(p)-T|
$$

where $I(p)$ is the intensity value in point $p$ and $\epsilon$ and $T$ specify the target intensity range: $I(p)$ between $T-\epsilon$ and $T+\epsilon$ promotes the expansion of the surface, while the intensity out of the range encourages a shrinkage. 
Our proposed texture term $D_{\text {tex }}$ influences the surface motion by taking all the previously generated texture features into consideration: the surface is encouraged to expand to a point $p$ if a feature similarity condition is fulfilled for all the selected texture features, or to contract otherwise, as defined in:

$$
D_{\text {tex }}(p)= \begin{cases}v & \text { if } \forall m \in M_{\text {best }}: \mid \text { val }_{m}(p)-\bar{x}_{m} \mid \leq \theta \times \sigma_{m} \\ -v & \text { otherwise, }\end{cases}
$$

where $m$ is a texture feature in the selected set $M_{\text {best }}, \bar{x}_{m}$ and $\sigma_{m}$ are the feature mean and standard deviation in the initial surface, $\operatorname{val}_{m}(p)$ is the value of the texture feature $m$ in the point $p, v$ is a predefined constant value and $\theta$ is a user-defined parameter that denotes the term sensitivity.

The level set equation is solved with a GPU-accelerated implementation of the upwind scheme [8]. The simulation time is currently determined by specifying the desired number of iterations. Due to the numerical stability requirements of the method, during a single iteration each surface point can be moved only by one position.

\section{Experimental Results}

This section shows the experimental segmentation results of the proposed method. As the algorithm was mostly GPU-bound, the experiments were performed on workstations with three types of Nvidia graphics cards: GeForce GT630 (with 96 CUDA cores), Nvidia Quadro K2000 (384 CUDA cores) and GeForce GTX780 (2304 cores). The total segmentation time for the presented examples is presented in Table 1. In comparison to the intensity-based level set method (see Eq. (2)), the proposed algorithm was about 2 times slower (on average). Each experiment consisted of 1000 iterations of the level set method.

The algorithm was implemented using the MESA system [11] - a platform for designing and evaluation of the deformable model-based segmentation methods. MESA provides a template system for construction of active contours from exchangeable elements (i.e., models, energies and extensions), allowing an easy comparison of the proposed approach with other methods. The GPU-accelerated algorithms were

Table 1 Segmentation time of the proposed method on different GPUs (in seconds)

\begin{tabular}{l|l|l|l}
\hline \multirow{2}{*}{ Data set } & GPU type & Quadro K2000 & GF GTX780 \\
\cline { 2 - 4 } & GF GT630 & 12 & 3.5 \\
\hline Volume 1 & 38 & 42 & 9 \\
\hline Volume 2 & 120 & & \\
\hline
\end{tabular}


implemented in OpenCL [16] and integrated with the existing code using a Java binding library (JOCL from www.jocl.org).

The method was tested on synthetic volumes, created using the Brodatz texture database [1]. The initial surfaces were initialized manually. The experiments usually required an adjustment of the $\theta$ and $\alpha$ parameters.

The first example (see Fig. 1) shows a segmentation of a synthetic volume $(256 \times$ $256 \times 9$ voxels) with an ellipsoidal region of a high contrast texture. The intensity variance of the pattern makes it impossible to segment with default intensity-based speed function (see Fig. 1c). The texture-based speed function, however, managed to correctly drive the surface to the region boundaries (see Fig. 1d).

The second example (see Fig. 2) presents a more challenging case: a volume $(256 \times 256 \times 20$ voxels $)$ containing a region with a complex surface and a texture identical to the background, but rotated by $90^{\circ}$. The default method failed to extract the region (see Fig. 2c). The texture-based method, however, managed to extract a rough outline of the surface due to the strong directional characteristics of the region pattern. The final form of the extracted surface is visible in Fig. 2f.
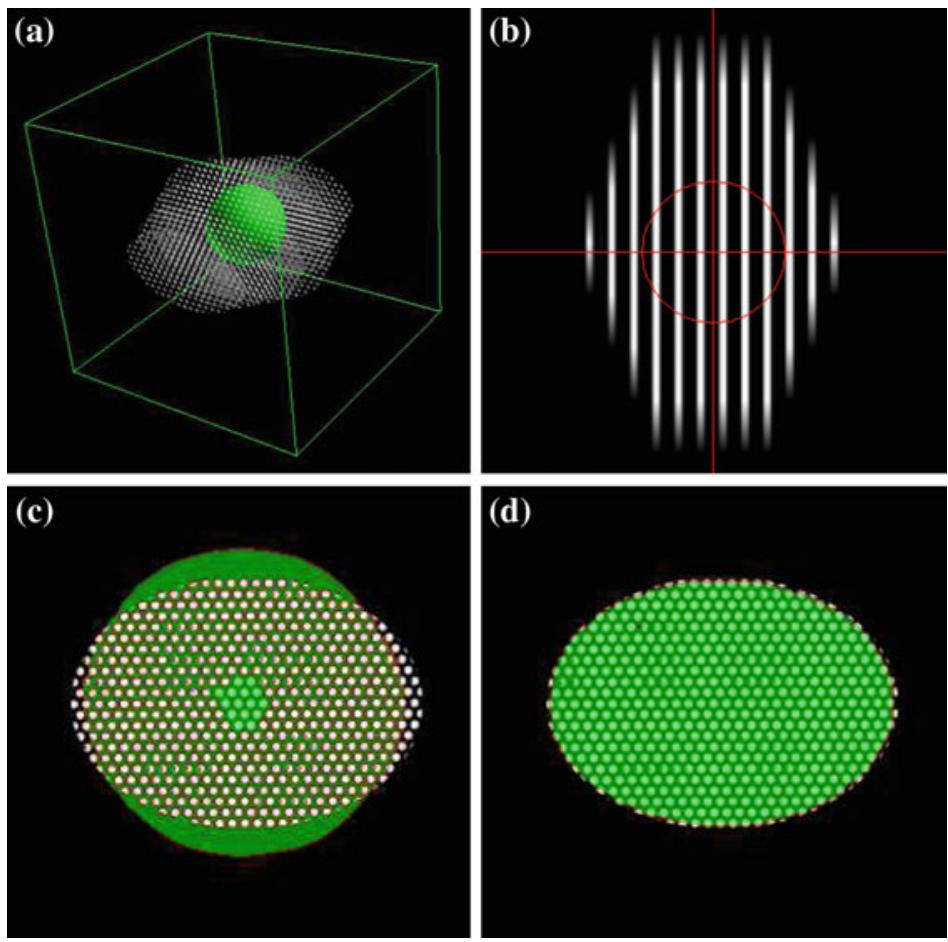

Fig. 1 Segmentation results of the first data sets: a 3D visualization of the set and initial surface, b initial surface in a $2 \mathrm{D}$ cross-section of the set, $\mathbf{c}$ sample result of the algorithm with the default image term, $\mathbf{d}$ result with the texture data term 

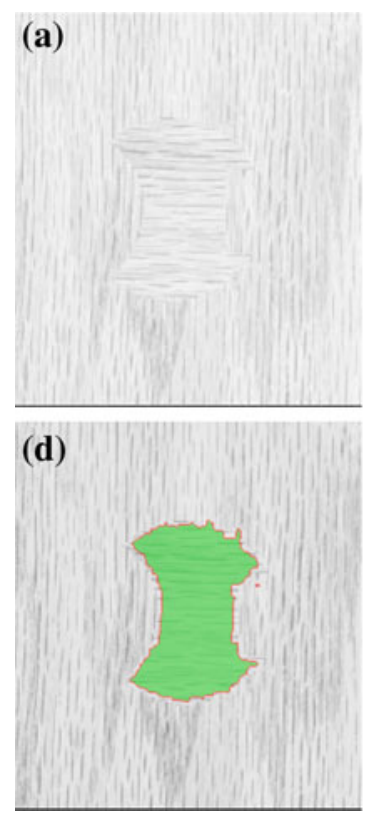
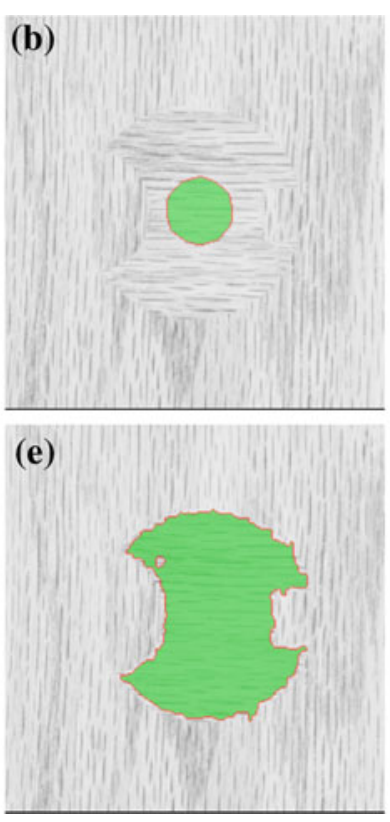
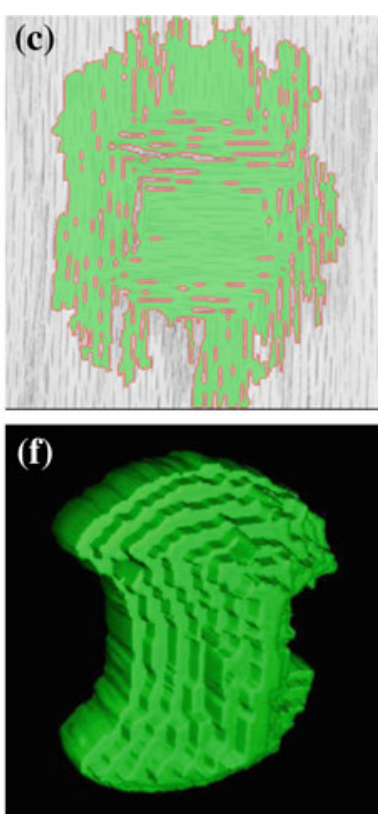

Fig. 2 Segmentation results of the second volume: a, b 2D slices from the volume with initialization visible on (b), c example volume slice with a result of the algorithm with the default image term, $\mathbf{d}$, e results with the texture data term, $\mathbf{f} 3 \mathrm{D}$ visualization of the extracted surface

\section{Conclusions and Future Work}

In this paper a texture-based level set method for segmentation of three-dimensional image volumes is proposed. The method implementation makes a heavy use GPU acceleration, achieved with OpenCL. While the present method is still in an early stage of development, the preliminary results on synthetic images show a potential for other applications, possibly in natural or medical image analysis.

In the future work, an important enhancement would be an utilization of fully 3D texture features [17] instead of the currently used combination of 2D textures. Moreover, the utilized feature set (based on GLCM) can be easily extended by incorporation of other texture feature extraction methods. Furthermore, while the current numerical level set solving method is relatively much faster than a CPU implementation, the nature of the method can suggest an employment of a different, more effective algorithm. As the surface is initialized inside the segmented region and is generally expected only to expand, a less computationally intensive method like fast marching [14] or narrow band [12] could be more suitable here.

Acknowledgments This work was supported by Bialystok University of Technology under Grant W/WI/5/2014 and S/WI/2/2013. 


\section{References}

1. Brodatz, P.: Textures: A Photographic Album for Artists and Designers. Dover Publications (1966)

2. Chan, T., Vese, L.: Active contours without edges. IEEE Trans. Image Proc. 10(2), 266-277 (2001)

3. Haralick, R., Shanmugam, K., Dinstein, I.: Textural features for image classification. IEEE Trans. Syst. Man Cybern. 6, 610-621 (1973)

4. Kass, M., Witkin, A., Terzopoulos, D.: Snakes: active contour models. Int. J. Comput. Vis. 1(4), 321-331 (1988)

5. Lefohn, A., Cates, J., Whitaker, R.: Interactive, GPU-based level sets for 3D segmentation. In: Medical Image Computing and Computer-Assisted Intervention-MICCAI 2003, pp. 564-572. Springer (2003)

6. Mcinerney, T., Terzopoulos, D.: T-snakes: Topology adaptive snakes. In: Medical Image Analysis, pp. 840-845 (1999)

7. Moore, P., Molloy, D.: A survey of computer-based deformable models. In: International Machine Vision and Image Processing Conference, IMVIP, pp. 55-66 (2007)

8. Osher, S., Sethian, J.: Fronts propagating with curvature-dependent speed: algorithms based on Hamilton-Jacobi formulations. J. Comput. Phys. 79(1), 12-49 (1988)

9. Paragios, N., Deriche, R.: Geodesic active regions and level set methods for supervised texture segmentation. Int. J. Comput. Vis. 46(3), 223-247 (2002)

10. Reska, D., Boldak, C., Kretowski, M.: A texture-based energy for active contour image segmentation. Adv. Intell. Syst. Comput. Image Process. Commun. Challenges 6(313), 187-194 (2015)

11. Reska, D., Jurczuk, K., Boldak, C., Kretowski, M.: MESA: complete approach for design and evaluation of segmentation methods using real and simulated tomographic images. Biocybernetics Biomed. Eng. 34, 146-158 (2014)

12. Roberts, M., Packer, J., Sousa, M., Mitchell, J.: A work-efficient GPU algorithm for level set segmentation. In: Conference on High Performance Graphics, pp. 123-132 (2010)

13. Ronfard, R.: Region-based strategies for active contour models. Int. J. Comput. Vis. 13(2), 229-251 (1994)

14. Sethian, J.: Level Set Methods and Fast Marching Methods: Evolving Interfaces in Computational Geometry, Fluid Mechanics, Computer Vision, and Materials Science, vol. 3. Cambridge University Press (1999)

15. Shen, T., Zhang, S., Huang, J., Huang, X., Metaxas, D.: Integrating shape and texture in 3D deformable models: from metamorphs to active volume models. In: Multi Modality Stateof-the-Art Medical Image Segmentation and Registration Methodologies, pp. 1-31. Springer (2011)

16. Stone, J., Gohara, D., Shi, G.: OpenCL: a parallel programming standard for heterogeneous computing systems. Comput. Sci. Eng. 12(3), 66 (2010)

17. Tesař, L., Shimizu, A., Smutek, D., Kobatake, H., Nawano, S.: Medical image analysis of 3D $\mathrm{CT}$ images based on extension of Haralick texture features. Comput. Med. Imaging Graph. 32(6), 513-520 (2008) 